\title{
Model Deformation Measurements of Sonic Boom Models in the NASA Ames 9- by 7-Ft Supersonic Wind Tunnel
}

\author{
Edward T. Schairer, ${ }^{1}$ Laura K. Kushner ${ }^{2}$, Theodore J. Garbeff ${ }^{3}$, and James T. Heineck ${ }^{4}$ \\ NASA Ames Research Center, Moffett Field, CA, 94035-1000
}

\begin{abstract}
The deformations of two sonic-boom models were measured by stereo photogrammetry during tests in the 9- by 7-Ft Supersonic Wind Tunnel at NASA Ames Research Center. The models were geometrically similar but one was 2.75 times as large as the other. Deformation measurements were made by simultaneously imaging the upper surfaces of the models from two directions by calibrated cameras that were mounted behind windows of the test section. Bending and twist were measured at discrete points using conventional circular targets that had been marked along the leading and trailing edges of the wings and tails. In addition, continuous distributions of bending and twist were measured from ink speckles that had been applied to the upper surfaces of the model. Measurements were made at wind-on $(M=$ 1.6) and wind-off conditions over a range of angles of attack between $2.5^{\circ}$ and $5.0^{\circ}$. At each condition, model deformation was determined by comparing the wind-off and wind-on coordinates of each measurement point after transforming the coordinates to reference coordinates tied to the model. The necessary transformations were determined by measuring the positions of a set of targets on the rigid center-body of the models whose model-axes coordinates were known. Smoothly varying bending and twist measurements were obtained at all conditions. Bending displacements increased in proportion to the square of the distance to the centerline. Maximum deflection of the wingtip of the larger model was about $5 \mathrm{~mm}$ (2\% of the semispan) and that of the smaller model was $0.9 \mathrm{~mm}(1 \%$ of the semispan). The change in wing twist due to bending increased in direct proportion to distance from the centerline and reached a (absolute) maximum of about $-1^{\circ}$ at the highest angle of attack for both models. The measurements easily resolved bending displacements as small as $0.05 \mathrm{~mm}$ and bending-induced changes in twist as small as $\mathbf{0 . 0 5}^{\circ}$.
\end{abstract}

\section{Introduction}

M odel deformation - the bending of a wind-tunnel model under aerodynamic loading - occurs during all windtunnel tests. In cases where the model is extremely stiff or the loads are very small (or both), the deformations may be negligible. In many tests, however, model deformation can be significant. The bending of wings is particularly important because it is likely to alter the twist distribution along the wing span and thus can affect the spanwise distribution of lift and the induced drag. If changes in model geometry are not measured, their effects can become confused with other sources of uncertainty such as wall interference and sub-scale Reynolds number. Not knowing the true geometry also makes comparisons of wind-tunnel data to computational fluid dynamic simulations more difficult.

Two optical techniques--photogrammetry and Moire interferometry--have been shown to be very effective methods for measuring model deformation. With photogrammetry, the three spatial coordinates of an object can be determined from images of the object acquired from at least two directions. The images must be acquired using calibrated cameras, i.e., cameras for which the transformation from object-space to the image-plane is known. If one of the space coordinates is known, then the other two coordinates can be determined from a single image.

Photogrammetry was first used to measure the deformation of a wind-tunnel model during tests in the 8-Ft Pressure Wind Tunnel at NASA Langley Research Center. ${ }^{1}$ In this test, targets were marked on the lower surface of a full-span, swept-wing model, and images of the model were recorded on film by two cameras mounted at windows

\footnotetext{
${ }^{1}$ Aerospace Engineer, Experimental Aero-Physics Branch, MS 260-1.

${ }^{2}$ Research Engineer, Aerospace Computing Incorporated/Experimental Aero-Physics Branch, MS 260-1.

${ }^{3}$ Instrumentation Engineer, Wind Tunnel Systems Branch, MS 227.

${ }^{4}$ InsePhotographic Technologist, Experimental Aero-Physics Branch, MS 260-1.
} 
in the floor of the test section. The image-plane positions of the targets on the films were measured with a moncomparator. The camera calibrations were based on the Direct Linear Transformation (DLT) approximation to the full colinearity equations (pinhole model) of photogrammetry. Targets on the fuselage and at the most inboard wing stations were assumed to be rigidly connected and were used to correct for model movement. The uncertainty of the wing bending measurements was $0.52 \mathrm{~mm}(0.13 \%$ of the semi-span), and the uncertainty of the twist measurements was $0.2^{\circ}$ at the wingtips.

The digital revolution that has occurred since these first measurements has enabled the development of automated photogrammetry systems that employ high-resolution digital cameras and powerful computers and produce model deformation measurements (MDM) in production wind tunnels while a test is in progress. Both commercial and custom (i.e., designed in-house) systems have been deployed in wind tunnels in Europe ${ }^{2-4}$ and the United States. ${ }^{5}$ In addition, model deformation measurements are sometimes included as part of the analysis of pressure-sensitive paint data. Except for the use of digital technology, contemporary MDM systems are remarkably similar to that first demonstrated at Langley. Most systems track the positions of discrete targets applied to the surfaces of the model and correct for model movement using a subset of rigidly connect targets. Some commercial systems facilitate automation by using coded targets or active, light-emitting, targets. ${ }^{6}$ One-camera Videometric Model Deformation (VMD) systems have been installed in several NASA wind tunnels. ${ }^{6-8}$ In these systems the need for a second camera is eliminated by assuming that the span-wise coordinates of the targets are known. Most contemporary systems make use of the full colinearity equations of photogrammetry, rather than the DLT approximation, and include corrections for lens distortion. The most accurate systems can measure twist in the laboratory with an uncertainty of only $0.01^{\circ}$. Uncertainties of measurements made in wind tunnels (a much less benign environment than the laboratory) with the NASA VMD systems are $0.1-0.2 \mathrm{~mm}$ in bending and $0.05^{\circ}$ in twist, although the twist uncertainty is highly dependent on the magnification.

The spatial density of measurements based on discrete targets is limited by the number and distribution of targets. Essentially continuous measurements can be made by covering the surface of the model with a random distribution of speckles rather than (or in addition to) targets. Any point on the model can be identified by the unique speckle pattern surrounding it. Image cross correlation, a technique commonly used in Particle Image Velocimetry ${ }^{9}$ (PIV), or optical flow ${ }^{10}$ is used to match small patches of speckles at each measurement point in the images of all cameras and in images acquired at wind-off and wind-on conditions. The spatial sampling is only limited by the density of the speckles and the resolution of the cameras. Of course, the speckles must not disturb the flow or change the shape of the model. A disadvantage of this method is that image cross correlation and optical flow are computationally intensive and take much longer than simply locating targets, which is very fast and easily automated. Speckle-based model-displacement measurements using commercial and custom photogrammetry systems ${ }^{11}$ have been reported. These include measurements of very flexible inflatable aerodynamic decelerators. ${ }^{12,13}$

Projection Moiré Interferometry (PMI) is an alternative to photogrammetry that also yields essentially continuous measurements of deformations normal to the surface of a model. ${ }^{5}$ A grid pattern is projected onto the model, and images are acquired at wind-off and wind-on conditions. Images of the projected grid are corrected for differences in perspective and "interfered" with a reference grid. This results in interferograms from which displacements normal to of the model surface can be derived. Like speckle photogrammetry, this analysis is computationally intensive. MDM systems based on PMI have been installed in large production wind tunnels at NASA $^{5}$ and in Europe. ${ }^{14,15}$

This paper describes deformation measurements that were made on the wing and tail surfaces of two scale models of the Boeing-NASA Quiet Experimental Validation Concept (QEVC) during tests in the 9- by 7-Ft

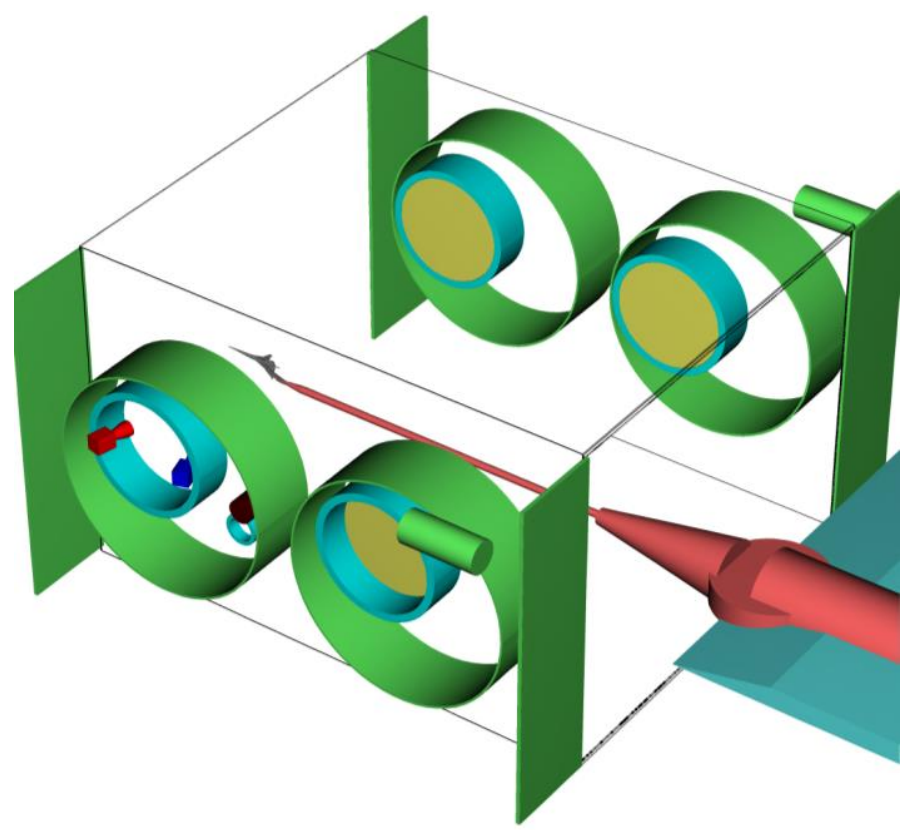

Figure 1. Schematic of 9- by 7-Ft test section. 
Supersonic Wind Tunnel at NASA Ames Research Center. These tests were part of the NASA High Speed Fundamental Aeronautics program. The measurements were made using a very-high resolution photogrammetry system that was developed in house. Deformation measurements were made using both speckles and conventional targets. To the best of our knowledge, these were the first model-deformation measurements to have been made in this wind tunnel.

\section{Apparatus}

\section{A. Wind Tunnel}

The tests were conducted in the 9- by 7-Ft Supersonic Wind Tunnel at NASA Ames Research Center. In this wind tunnel, Mach numbers in the test section between 1.5 and 2.5 are set by sliding the lower half of a twodimensional asymmetric nozzle in the streamwise direction, thereby altering the height of the sonic throat. The models were tested with their wings in a vertical plane, which is the usual configuration in this tunnel (Fig. 1). They were supported by a sting that extends upstream from a horizontal strut that spans the test section at its downstream end. Angle of attack of the wings-vertical models was set by pivoting the sting in the horizontal pitch plane about a "knuckle sleeve" at the leading edge of the strut. The strut and sting can be translated in the horizontal pitch plane to place the model at any cross-stream position.

Windows in the side-walls of the test section were designed to permit schlieren measurements. Two pairs of circular windows (28.5-in dia) can be mounted in corresponding pairs of turntables in the test section sidewalls, one pair nearer the upstream end and the other nearer the downstream end. The windows are mounted eccentrically in the turntables so that different portions of the test section can be illuminated by rotating the turntables. Three 6.25 -in diameter optical portholes were recently added to one of the upstream turntables. For the present tests, windows were installed only in the upstream turntable of the sidewall toward which the upper surfaces of the models faced (south).

\section{B. Models}

Two scale models of the Boeing-NASA Quiet Experimental Validation Concept (QEVC) were tested. One model was relatively small (wingspan $=171 \mathrm{~mm}, 0.65 \%$ of the full-scale configuration) and was used for measurements of sonic-boom pressure signatures. The second model was 2.75 times as large as the first (wingspan $=470 \mathrm{~mm}$ ) and was used for measurements of the performance of the configuration. Thus, the designations of the small and large models were "Boom" and "Performance," respectively. The wings of the models were swept and had winglets at the tips and leading-edge extensions at the root (Fig. 2). Two engine nacelles were located on the upper surface and at the downstream end of a broad center-body. Two tail surfaces were located outboard of the nacelles and were canted outboard to form a "V." The models were constructed of Vascomax C-300 steel. Though the models were geometrically similar, their structures were different. The wings and the center-body of the Boom

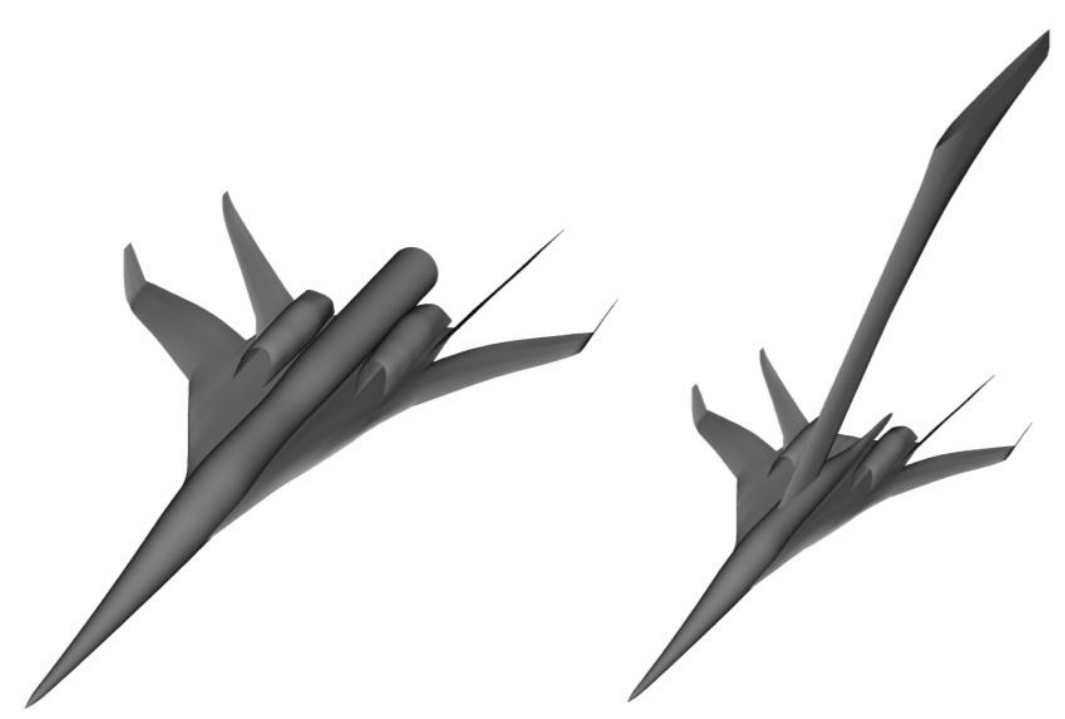

Figure 2. Performance model (left) and Boom model (right). model were constructed from a single, solid, piece of material. Each wing of the Performance model was a separate piece of material that was pinned to the center-body through a tongue-in-groove joint.

The model supports were also different. The Boom model was attached to the sting by a swept blade that extended from the upper surface of the center-body (Fig. 2). With this arrangement the centerline of the model was offset below (in model axes) the centerline of the sting. The Performance model was attached to the sting by a cylindrical member that extended downstream from the aft end of the center-body and was coaxial with the sting. With these supports, the wings of the Performance model 

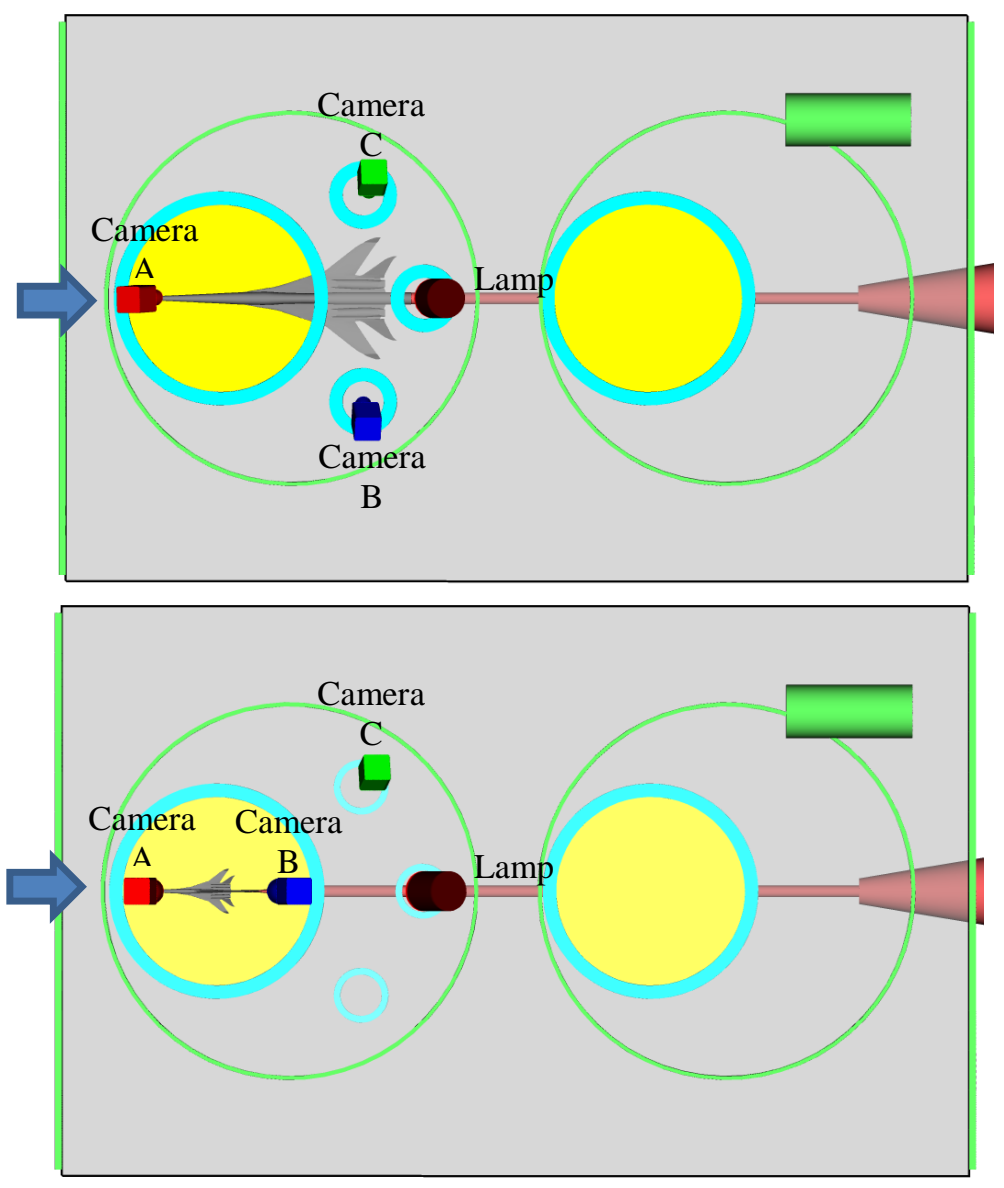

Figure 3. Side views of test section showing models and placement of cameras and lamp. were nearly three feet further downstream than the wings of the Boom model (Fig.3).

Model deformation measurements were made using two types of targets: conventional circular targets that were marked on the upper surface of the wings along the leading and trailing edges; and high-density targets formed by random, very fine, speckles applied to the upper surfaces of the wings and tail. In addition, model features on the fuselage such as the heads of small screws were used as targets for computing rigid-body displacements.

The speckle targets were created by first painting the upper surface of the model with black inkjet ink using a small artist's airbrush (Fig. 5). The airbrush was adjusted to produce a very fine spray that resulted in a continuous sheet of tiny speckles. After this black coat had dried, the procedure was repeated, but this time using a lighter coat of white ink. Together, these coats produced a matte, high-contrast, dense speckle distribution. After the ink had dried, it was smoothed by vigorously wiping it with cheese cloth. No quantitative measurements of the surface roughness were made; however, after it had been smoothed, the speckled surface felt to the touch about as smooth as the bare metal. The conventional targets were drawn on top of the speckles using a black permanent marker. The

targets were about $1 \mathrm{~mm}$ in diameter, and they were not perfectly round because they were drawn free-hand without a template. Contrast between the targets and the speckled background was poor. This greatly increased the uncertainty in locating the target centroids.

\section{Instrumentation}

The models were imaged by three Nikon D-800 35-megapixels cameras that viewed the upper surfaces of the models through the windows in the south sidewall of the test section (Fig. 3). Because the models were tested with the wings vertical with upper surfaces facing south, all cameras had a good view of the upper surfaces. The upstream turntable was rotated so that the schlieren window was at its most upstream position. For the performance model, one camera (A) was located at the upstream edge of the schlieren window and was pointed slightly downstream. The other two cameras (B and $\mathrm{C}$ ) were placed further downstream and viewed the model through the portholes in the turntable above and below the tunnel centerline. For the model deformation measurements the models were displaced approximately 18 in $(45.7 \mathrm{~cm}$ ) toward the south wall (and cameras) from the plane midway between the north and south walls. This was done to increase the difference in viewing angles (parallax) between the cameras.

For measurements of the performance model, all three cameras were fitted with $85 \mathrm{~mm}$ tilt-shift lenses that could be rotated and tilted to provide good edge-to-edge focus (scheimpflug). The field of view of each camera included only the starboard half of the model. For measurements of the smaller boom model, $135 \mathrm{~mm}$ lenses without tilt capability were installed. In addition, camera B was moved upstream from the lower-downstream porthole to the downstream edge the schlieren window where its view of the model was less oblique (Fig. 3). The field of view of each camera included both the port and starboard halves of the boom model.

The lens apertures were set to f/16. The model was illuminated by a Dyna-Lite flash-lamp that was placed in the center-downstream porthole of the upstream turntable and was triggered from the hot shoe of one camera. The flash 

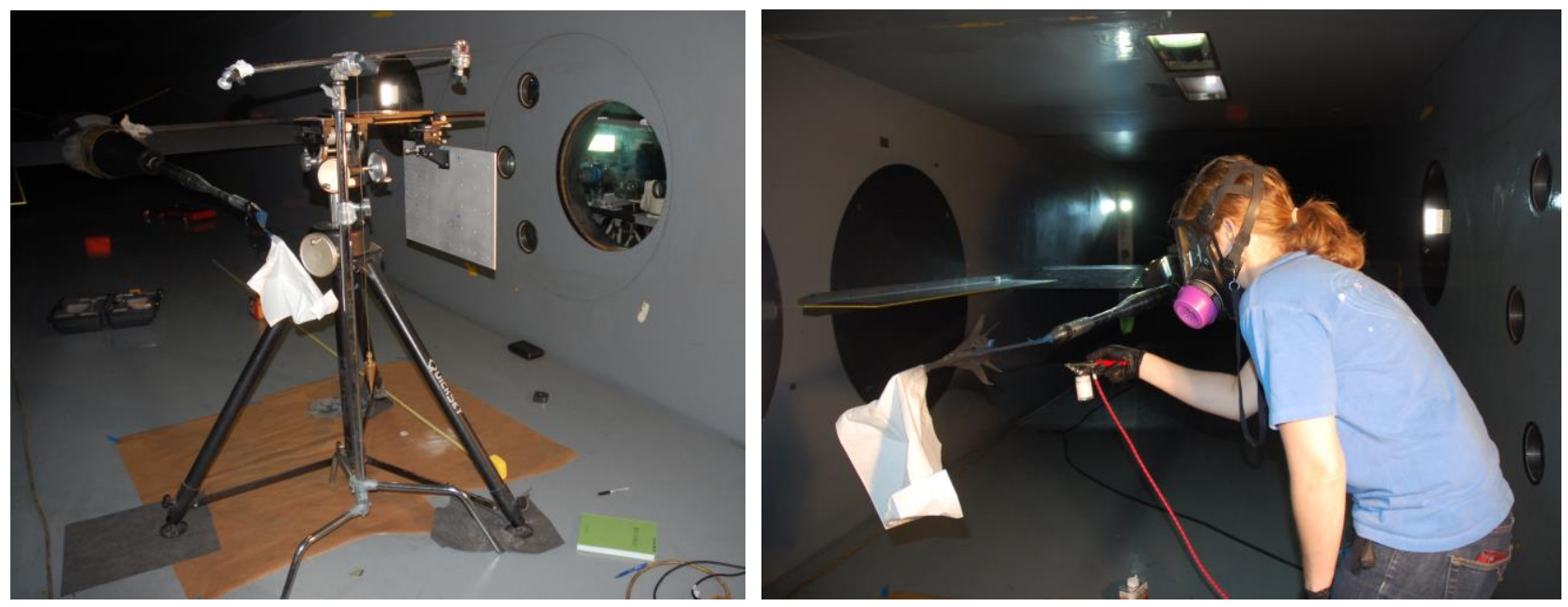

Figure 4. Calibration plate installed in test section. Figure 5. Applying speckles to Performance model.

was timed to occur after the camera shutters had opened, and the flash duration $(0.5-0.7 \mathrm{msec})$ was much shorter than the time the camera shutters were open. Therefore, since there were no other significant light sources, the flash synchronized the camera exposures and established the effective integration time.

The cameras were calibrated in situ by placing a vertical flat plate in the volume occupied by the model. The plate, which included a rectangular array of holes spaced at precise intervals of $20 \mathrm{~mm}$, was aligned parallel to the sidewalls and was imaged by all cameras at several lateral positions that bracketed the positions of the model (Fig. 4). The distance between extreme lateral positions - about 6 inches - was too large to allow the plate to be moved using a micrometer-driven translation stage. Therefore, the plate was re-aligned to the test section at each position using a laser level and a plumb bob.

\section{Data Acquisition}

Image data were acquired using two LABview scripts that triggered the cameras and flash lamp simultaneously. The first script was used to acquire images of the Performance model. It was convenient because it automatically acquired, downloaded, and renamed the images with run and sequence numbers. The time required to download the images (100 Mb each), however, was excessive and severely limited the rate at which data could be acquired. Therefore, only ten images were acquired at each condition. The second script was used for the Boom model and simply acquired the images and left them on the cameras' internal CF cards. Because no time was lost downloading images, more images - 40 - were acquired at each test condition. The images were retrieved from the cameras after the test. A series of wind-off images was acquired after the wind-on runs at the same angles as those at which the wind-on images had been acquired.

\section{Data Reduction}

The MDM images were analyzed using photogrammetry software that was developed in house. The analysis was based on the Direct Linear Transformation ${ }^{16}$ approximation to the full collinearity equations ${ }^{17}$ of photogrammetry:

$$
\begin{gathered}
X=\frac{L_{1} x+L_{2} y+L_{3} z+L_{4}}{L_{9} x+L_{10} y+L_{11} z+1}+\Delta X_{\text {lens }} \\
Y=\frac{L_{5} x+L_{6} y+L_{7} z+L_{8}}{L_{9} x+L_{10} y+L_{11} z+1}+\Delta Y_{\text {lens }}
\end{gathered}
$$

where $(x, y, z)$ and $(X, Y)$ are the object-space and image-plane coordinates of a point, respectively; $\Delta X_{\text {lens }}$ and $\Delta Y_{\text {lens }}$ are nonlinear terms that correct for lens distortion; and $L_{L}-L_{11}$ are coefficients determined by calibration. For the present test the nonlinear corrections $\Delta X_{\text {lens }}$ and $\Delta Y_{\text {lens }}$ were very small and were not included. 
The coefficients $L_{1}-L_{11}$ were determined from images of the calibration plate. If the object-space $\left(x_{i}, y_{i}, z_{i}\right)$ imageplane $\left(X_{i}, Y_{i}\right)$ coordinates of $n$ calibration targets are known, then the coefficients $L_{1}-L_{11}$ can be computed by solving an over-determined set of linear equations:

$$
\left[\begin{array}{ccccccccccc}
x_{1} & y_{1} & z_{1} & 1 & 0 & 0 & 0 & 0 & X_{1} x_{1} & X_{1} y_{1} & X_{1} z_{1} \\
x_{2} & y_{2} & z_{2} & 1 & 0 & 0 & 0 & 0 & X_{2} x_{2} & X_{2} y_{2} & X_{2} z_{2} \\
\vdots & \vdots & \vdots & \vdots & \vdots & \vdots & \vdots & \vdots & \vdots & \vdots & \vdots \\
x_{n} & y_{n} & z_{n} & 1 & 0 & 0 & 0 & 0 & X_{n} x_{n} & X_{n} y_{n} & X_{n} z_{n} \\
0 & 0 & 0 & 0 & x_{1} & y_{1} & z_{1} & 1 & Y_{1} x_{1} & Y_{1} y_{1} & Y_{1} z_{1} \\
0 & 0 & 0 & 0 & x_{2} & y_{2} & z_{2} & 1 & Y_{2} x_{2} & Y_{2} y_{2} & Y_{2} z_{2} \\
\vdots & \vdots & \vdots & \vdots & \vdots & \vdots & \vdots & \vdots & \vdots & \vdots & \vdots \\
0 & 0 & 0 & 0 & x_{n} & y_{n} & z_{n} & 1 & Y_{n} x_{n} & Y_{n} y_{n} & Y_{n} z_{n}
\end{array}\right] \times\left[\begin{array}{c}
L_{1} \\
L_{2} \\
L_{3} \\
L_{4} \\
L_{5} \\
L_{6} \\
\vdots \\
L_{11}
\end{array}\right]=\left[\begin{array}{c}
-X_{1} \\
-X_{2} \\
\vdots \\
-X_{n} \\
-Y_{1} \\
-Y_{2} \\
\vdots \\
-Y_{n}
\end{array}\right]
$$

There must be at least six calibration targets that do not all lie in the same plane. This equation is usually illconditioned, but it can be solved in a least-squares sense by singular value decomposition. ${ }^{18}$

The space coordinates $(x, y, z)$ of any point that appears in the images of two or more calibrated cameras $(A, B$, $\ldots, N)$ can be computed from:

$$
\left[\begin{array}{ccc}
X^{A} L_{9}^{A}-L_{1}^{A} & X^{A} L_{10}^{A}-L_{2}^{A} & X^{A} L_{11}^{A}-L_{3}^{A} \\
Y^{A} L_{9}^{A}-L_{5}^{A} & Y^{A} L_{10}^{A}-L_{6}^{A} & Y^{A} L_{11}^{A}-L_{7}^{A} \\
X^{B} L_{9}^{B}-L_{1}^{B} & X^{B} L_{10}^{B}-L_{2}^{B} & X^{B} L_{11}^{B}-L_{3}^{B} \\
Y^{B} L_{9}^{B}-L_{5}^{B} & Y^{B} L_{10}^{B}-L_{6}^{B} & Y^{B} L_{11}^{B}-L_{7}^{B} \\
\vdots & \vdots & \vdots \\
X^{N} L_{9}^{N}-L_{1}^{N} & X^{N} L_{10}^{N}-L_{2}^{N} & X^{N} L_{11}^{N}-L_{3}^{N} \\
Y^{N} L_{9}^{N}-L_{5}^{N} & Y^{N} L_{10}^{N}-L_{6}^{N} & Y^{N} L_{11}^{N}-L_{7}^{N}
\end{array}\right] \times\left[\begin{array}{c}
X \\
y \\
Z
\end{array}\right]=\left[\begin{array}{c}
L_{4}^{A}-X^{A} \\
L_{8}^{A}-Y^{A} \\
L_{4}^{B}-X^{B} \\
L_{8}^{B}-Y^{B} \\
\vdots \\
L_{4}^{N}-X^{N} \\
L_{8}^{N}-Y^{N}
\end{array}\right]
$$

$\left(X^{A}, Y^{A}\right),\left(X^{B}, Y^{B}\right), \ldots,\left(X^{N}, Y^{N}\right)$ are the image-plane coordinates of the target in images $A-N$. It is critical that they are accurately located in the images and that they correspond to the same point in space ("correspondence"). Like Eq. 2, this is an over-determined problem with an approximate, least-squares solution. In general, the approximation improves as the number of cameras increases (the least-squares error decreases as $1 / \sqrt{N}$ ).

If the model were supported absolutely rigidly and at exactly the same angle at wind-off and wind-on conditions, then model deformation could be simply determined by locating the a set of targets in the images of all cameras at both wind-off and wind-on conditions, computing the object-space coordinates of these targets from Eq 3, and forming the difference. In the present tests, however, the models were supported at the ends of long stings and experienced considerable movement due to unsteady air loads. In addition, the angles of attack at wind-off and wind-on conditions did not match exactly. Therefore, it was necessary to separate differences between wind-off and wind-on coordinates into contributions due to rigid-body movement and contributions due to elastic deformation. This separation was accomplished by tracking a set of targets on the center-bodies of the models that were assumed to be rigidly connected. The space coordinates of these targets in body-axes coordinates $\left(\alpha=0^{\circ}\right)$ were known (supplied by Boeing), and their instantaneous coordinates in tunnel coordinates were measured by photogrammetry. Then the measured coordinates were transformed as a rigid body to give the best fit to the true, body-axes, coordinates. This is an over-determined, non-linear, least-squares problem that was solved by the LevenbergMarquardt method. ${ }^{19}$ Rigid-body transformations were computed from the wind-off images and at each wind-on instance and were applied to the space coordinates of all measurement points, including those on flexible parts of the models. This procedure brought all points at both wind-off and wind-on conditions into the body-axes coordinate system where remaining differences were due to model deformation.

The rigid-body targets for the Performance model were the heads of screws and other features on the model's center-body. For the Boom model there were not enough well-defined, easily identifiable, features on the centerbody to allow computing an accurate rigid-body transformation. Therefore, an alternate method was employed, as discussed below.

Measurement points on flexible portions of the models were defined in two ways: using conventional targets marked along the leading and trailing edges of the wings and tails; and, for the speckle method, by mapping a CFD surface grid for the model (supplied by Boeing) to the wind-off images. The conventional targets were identified manually in the wind-off images and in the first wind-on image from each camera using the computer mouse to 
point and click. Targets in subsequent wind-on images were automatically located by cross correlation with the first wind-on image.

The surface grid used for the speckle method was a structured, rectangular grid with separate zones for the upper surfaces of the wing (75x75 nodes) and tail (50x50 nodes) and was expressed in body-axis coordinates. The grid was transformed to tunnel axes before mapping it to the images by applying the inverse of the rigid-body transformation described above. Then the image coordinates of each node were computed from Eq. 1.

Each node of the grid was uniquely identified by the random speckles surrounding it. For perfect mappings, each node would overlie the same speckles in the images from all cameras and correspondence would be satisfied. In practice, however, the speckles at each node did not match exactly. To correct for this error, the position of each node in the wind-off images from the second and third cameras (B and C) was adjusted so that it overlay the same speckles as in the wind-off image from the first camera (A) (i.e., correspondence was enforced). The required displacements were computed by first "dewarping" the image data at each node to minimize differences in perspective, and then cross correlating the dewarped image data from cameras B and C with data from camera A at each node.

Wind-off images acquired at the lowest angle of attack were used as references for wind-on images acquired at all angles of attack. This assured that the measurement points (local speckles) were the same at all angles.

Once correspondence of nodes was established in the wind-off images from all cameras, no further camera-tocamera cross correlation was required, and the positions of the nodes in the wind-on images from each camera were tracked independently. The mapping of the surface grid to the wind-on images always included a small error because the grid represented the un-deformed shape of the model. This was acceptable because the measurement points (unique speckles) were defined in the wind-off images. The wind-on mapping needed only to place each node in the neighborhood of the speckles defined in the wind-off images. Then, the displacement of each node (speckle pattern) in each wind-on image relative to its position in the wind-off image was computed by wind-on -to- windoff image cross correlation within a small interrogation window centered on the node. By repeating this procedure at all nodes of each wind-on image, the wind-on image coordinates of the surface grid were defined in the images of all cameras.

The object-space coordinates of each point were computed from the point's coordinates in the images from all cameras using Eq. 3. The measurements at each instance were transformed to body axes by applying translations and rotations computed from the rigid-body targets. Separate transformations were applied to the wind-off coordinates and to the wind-on coordinates at each instance. The remaining differences between wind-on and wind-off coordinates of the measurement points were assumed to be due to model deformation. Two quantities were of particular interest: displacements perpendicular to the plane of the wing or tail (bending) and changes in the local twist of the wing or tail due to model deformation. The change in twist at each span station (i.e., along each row of the surface grid) was determined by fitting the vertical displacement data along the chord with a straight line. The slope of this line, in degrees, was the deformation-induced twist (negative if the trailing-edge displacement exceeded the leading edge displacement). Twist measurements using conventional targets were based on only two targets at each span station - at the leading and trailing edges.

For speckle-based measurements of the Boom model, there were too few clearly identifiable targets on the center-body to allow computing an accurate rigid-body transformation. Therefore, the procedure described above was modified. First, the tips of the tails, which were widely separated and could be accurately located in the images, were included as rigid-body targets for the purpose of mapping the surface grid to the images. Including the tail tips yielded accurate mappings at wind-off conditions because the tail was not loaded and did not deflect. At wind-on conditions, however, the deflection of the tail introduced small mapping errors. Wind-on mapping errors occurred anyway and, as discussed above, were acceptable as long as they were small. However, errors in the wind-on rigidbody transformation could not be tolerated when wind-on measurements were transformed to body axes. Therefore, the transformation to body axes was computed using measured (tunnel-axes) and actual (body-axes) coordinates of the most inboard row of nodes of the grid on both wings. This procedure effectively imposed a zero-bending constraint along the roots of both wings.

Angle of attack $(\alpha)$ was a by-product of the rigid-body transformation from tunnel to body-axis coordinates and was also measured by the wind-tunnel data system (SDS). Measuring $\alpha$ has always been problematic in the 9- by 7Ft Wind Tunnel because the pitch plane is usually horizontal, so gravity-based methods cannot be used. Instead, the SDS angle of attack is determined using data from encoder/resolvers in the knuckle-sleeve and other elements of the model support system. In addition, corrections for model deflection can be computed from loads measured by the balance, but these corrections were not applied in the present test. Uncertainties in angles measured by SDS are not reported. 


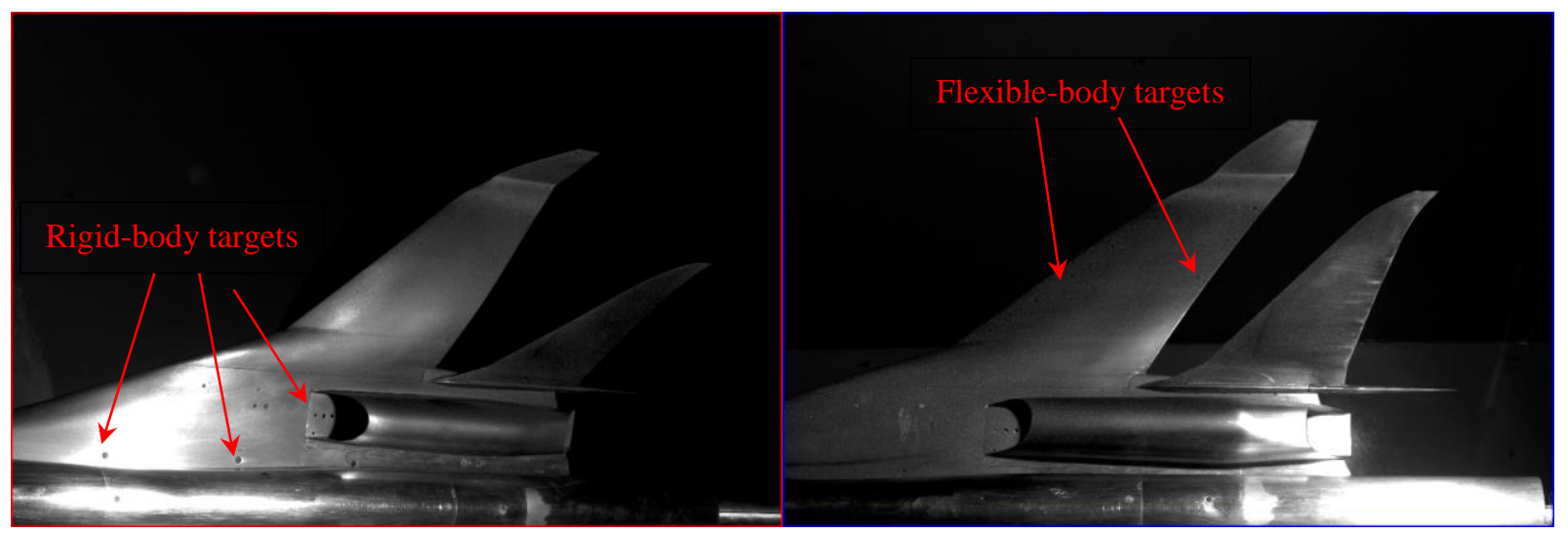

Figure 6. Images of Performance Model from upstream (left) and lower-downstream (right) cameras.

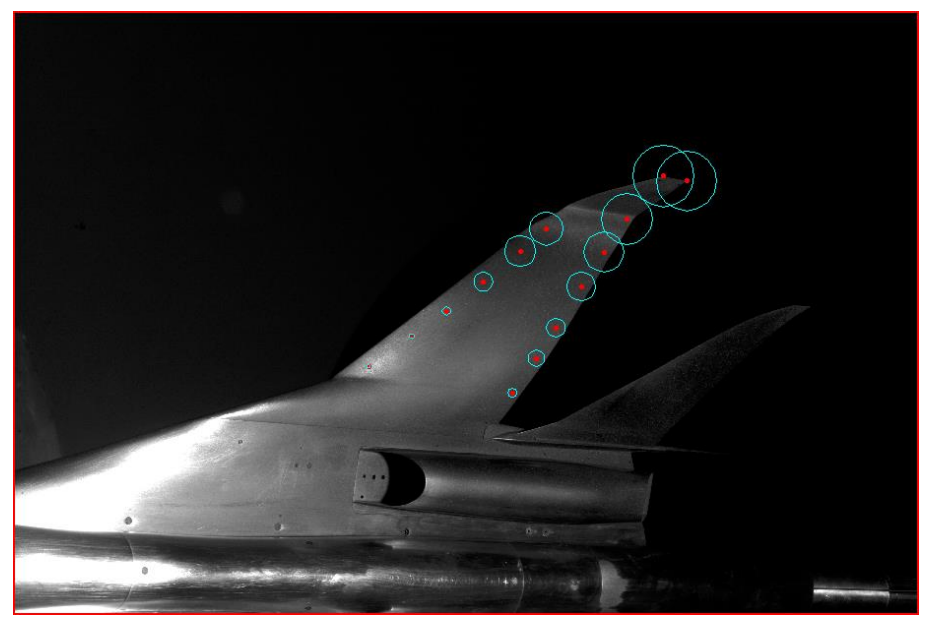

Figure 7. Bending displacements of flexible-body targets on wing of Performance model.
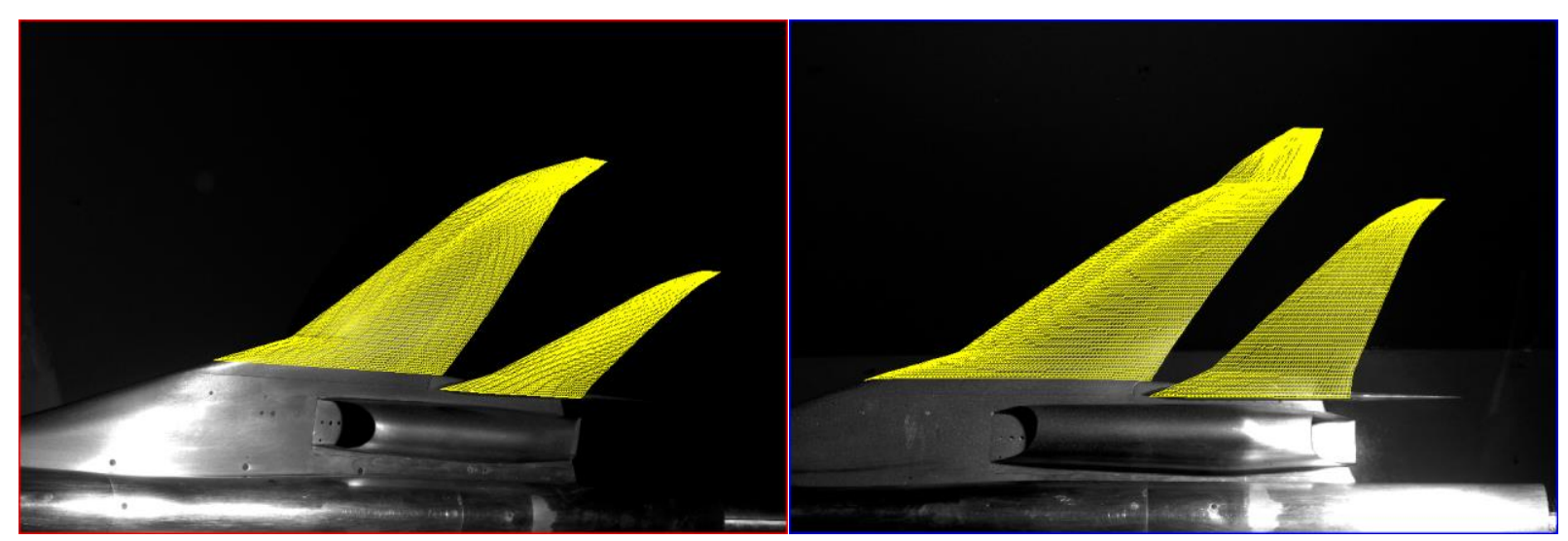

Figure 8. Images of Performance model from upstream (left) and downstream (right) cameras showing surface grid.

American Institute of Aeronautics and Astronautics 

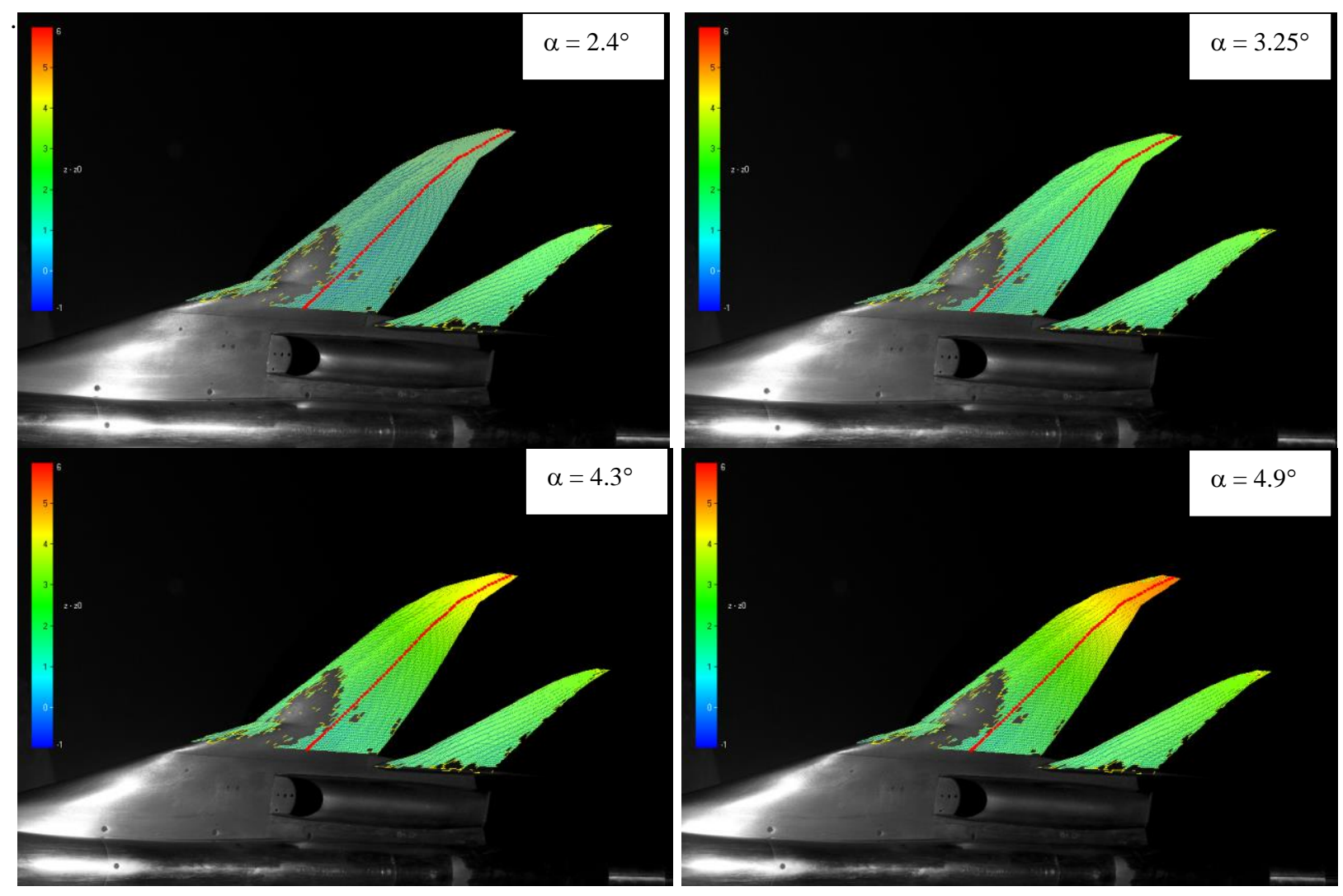

Figure 9. Images of Performance model at different angles of attack with overlay of bending displacements. Color scale maximum is $6 \mathbf{~ m m}$ (red) and minimum is $\mathbf{- 1} \mathbf{~ m m}$ (blue).
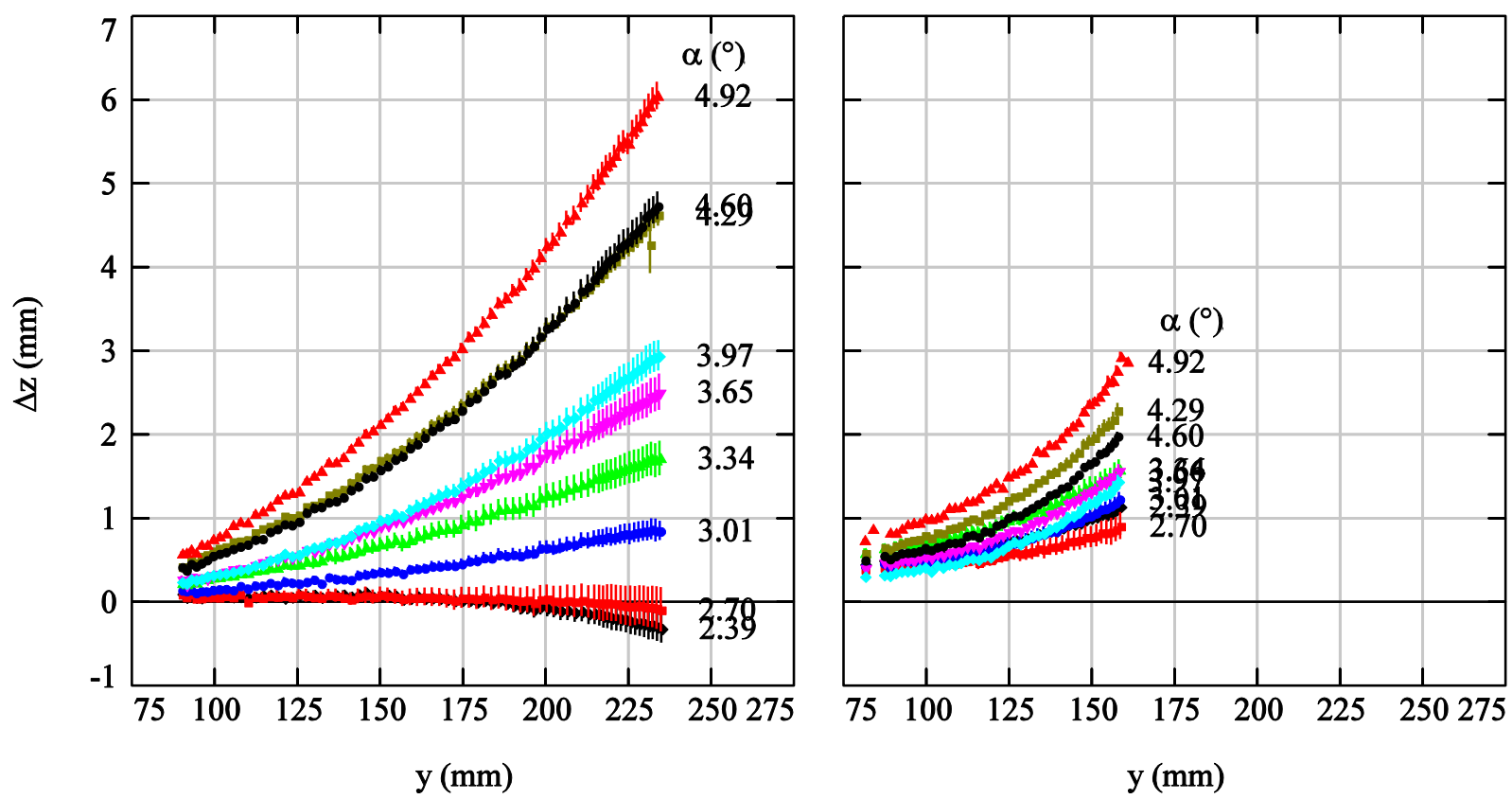

Figure 10. Bending displacements of the Performance model along spanwise line of wing (left) anf tail (right).

9

American Institute of Aeronautics and Astronautics 

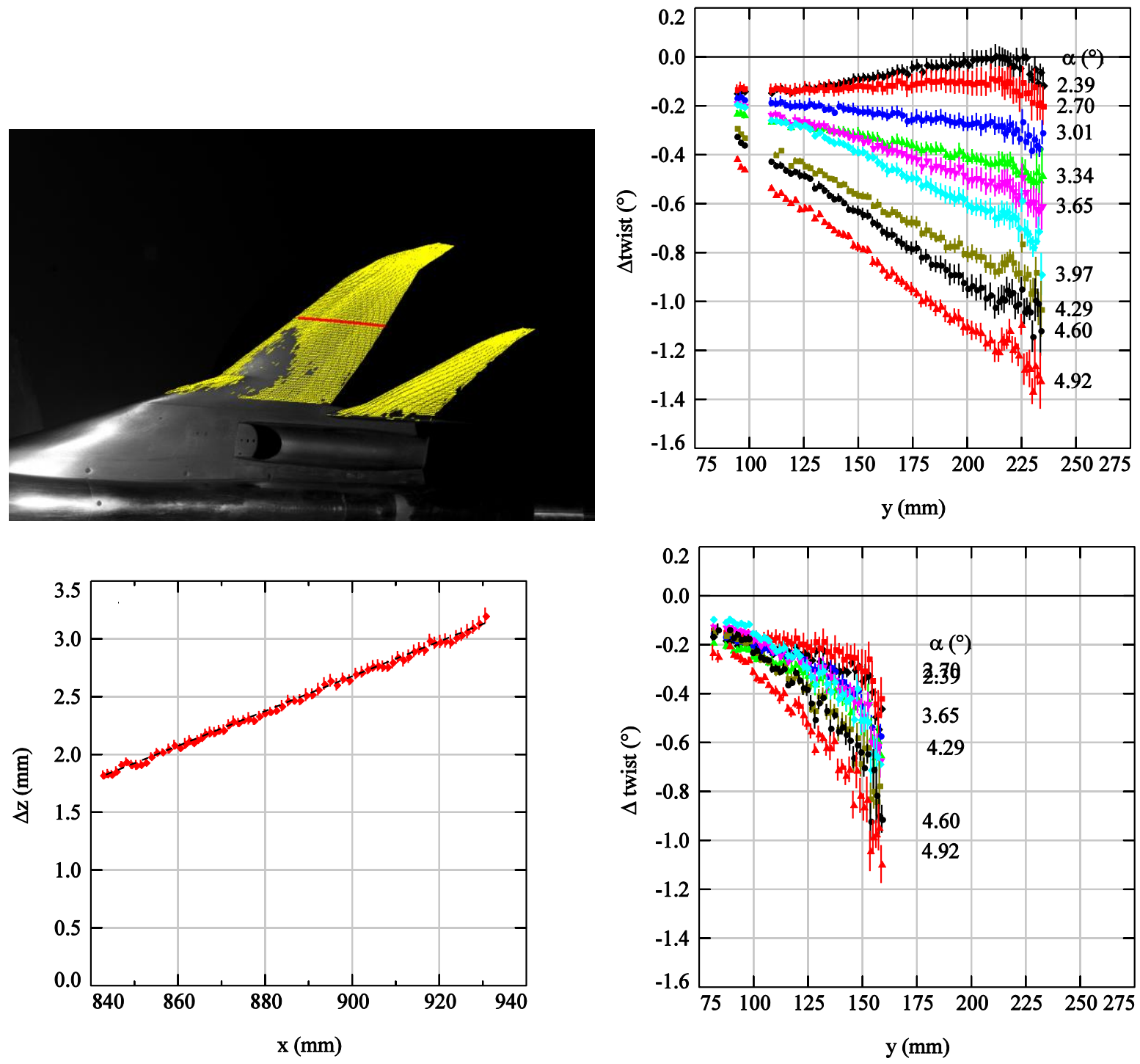

Figure 11. Twist due to bending of Performance model. Bending displacements along the chord-wise line at top-left are plotted at bottom-left. The slope of the best linear fit is the local change in twist. Changes in twist vs span are shown for wing (top-right) and tail (bottom-right).

body targets along the leading and trailing edges are barely visible in both images

Figure 7 shows average bending measurements at the maximum $\left(\alpha=4.92^{\circ}\right)$ angle of attack computed using data at the targets. Wind-on images were acquired at 10 instances. At each instance solid-body rotation/translation were subtracted, then the total deflection was computed and averaged. Circle size indicates the amount of out-of-plane bending at each target, and color its direction, where red is into the page and blue is out of the page.

Figure 8 shows wind-off images of the Performance model from the upstream (A) and lower-downstream (B) cameras with the surface grid that was projected into both images. Figure 9 shows a sequence of wind-off images where the wind-on bending of the wing and tail at increasing angles of attack are indicated by color overlays. Data are missing from a region on the wing because of a "hot spot" in the images from the upstream camera where speckle contrast was very low (see Fig. 6). Figure 10 shows the wing and tail bending for all cases along the red span-wise lines shown in Figure 9. These data are the average of ten instantaneous measurements. Bending displacements increased with angle of attack, and, at each angle, the displacements along the span increased with the square of the distance from the centerline. 

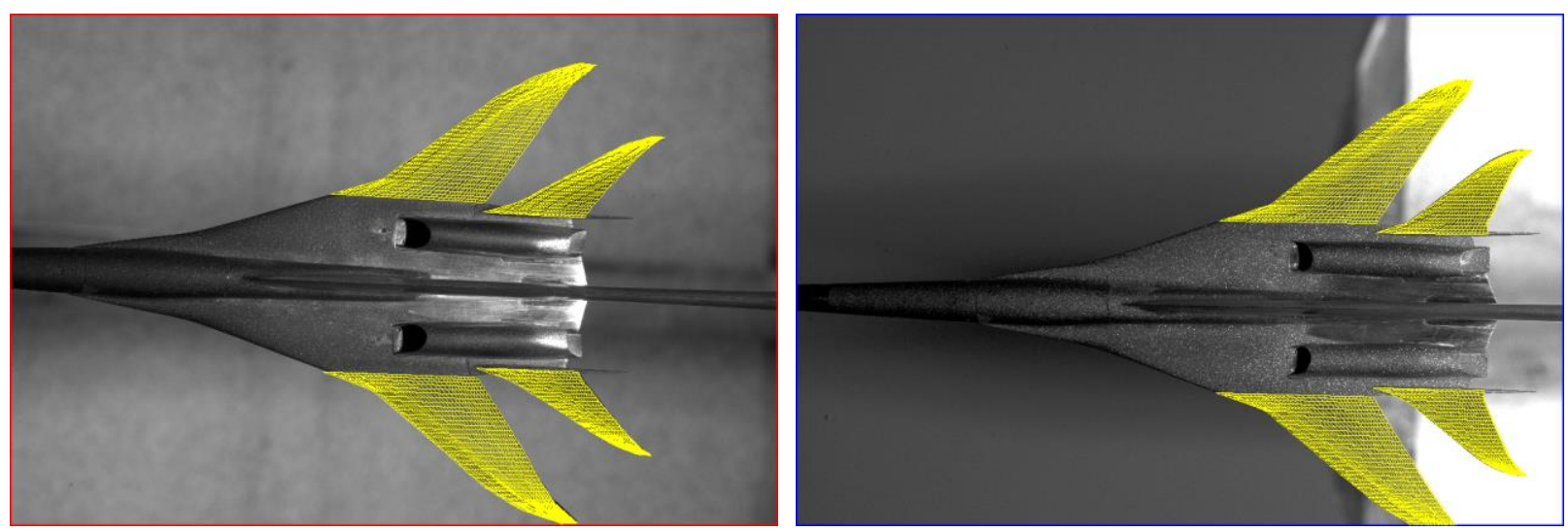

Figure 12. Images of Boom model from upstream (left) and downstream (right) cameras with grid overlay.

Figure 11 shows changes in twist due to bending for the Performance model The graph at lower-left shows bending along the chordwise line shown in the image at top-left. The slope of the line that best fits these data is the bending-induced twist at that span station. Bending-induced twist versus span for the wing and tail are shown at topright and bottom-right, respectively. The twist becomes increasingly negative as angle of attack and bending increase. At the two lowest angles, where the wing bends down rather than up, the direction of twist also changes sign. The variation of twist versus span is very linear at all angles of attack for the wing but is much less linear for the tail.

\section{B. Boom Model}

Figure 12 shows wind-off images of the Boom model from the upstream and downstream cameras with the surface grid superimposed. Both the port and starboard sides of the Boom model were included in the images.

Figure 13 shows bending data for the boom model superimposed on wind-off images. Bending increased with angle of attack, and there was good left-right symmetry at all angles. Average bending along both wings and tails are plotted versus spanwise position in Figure 14. As for the performance model, the wing bending at the two lowest angles of attack was in the opposite direction as bending at the higher angles. Bi-lateral symmetry was good. Finally, Figure 15 shows average bending-induced twist versus span on the wing and tail. The data show that washout increases with distance outboard and angle of attack.

\section{Angle of Attack}

Figure 16 compares angles of attack computed from MDM to angles of attack measured by SDS. The MDM data are averages of instantaneous measurements - 10 at each angle for the Performance model, and 40 for the Boom model. The MDM angles of the Performance model were uniformly slightly higher than the angles reported by SDS. The average difference was $0.14^{\circ}$ and the standard deviation of the difference was $0.056^{\circ}$. After eliminating the average offset, the RMS difference between the measurements was $0.046^{\circ}$. For the Boom model, the MDM angles were uniformly less than those of SDS. The average difference was $0.51^{\circ}$ with a standard deviation of $0.0035^{\circ}$. After eliminating the average offset, the RMS difference was $0.0086^{\circ}$.

\section{Discussion}

\section{A. Uncertainty}

Many factors contributed to the uncertainty of the MDM measurements. The most important of these were uncertainty in the alignment of the calibration plate, which produces a bias error, and random errors in locating corresponding measurement points in the images of both cameras. Other less important sources of uncertainty were calibration errors due to uncertainty in locating targets in images of the calibration plate; calibration errors due to vibration-induced camera movement; errors in the DLT approximation of the relationship between image and object space (Eq. 1); and errors due to the inexact least-squares solution of space coordinates from image coordinates (Eq. $3)$. 

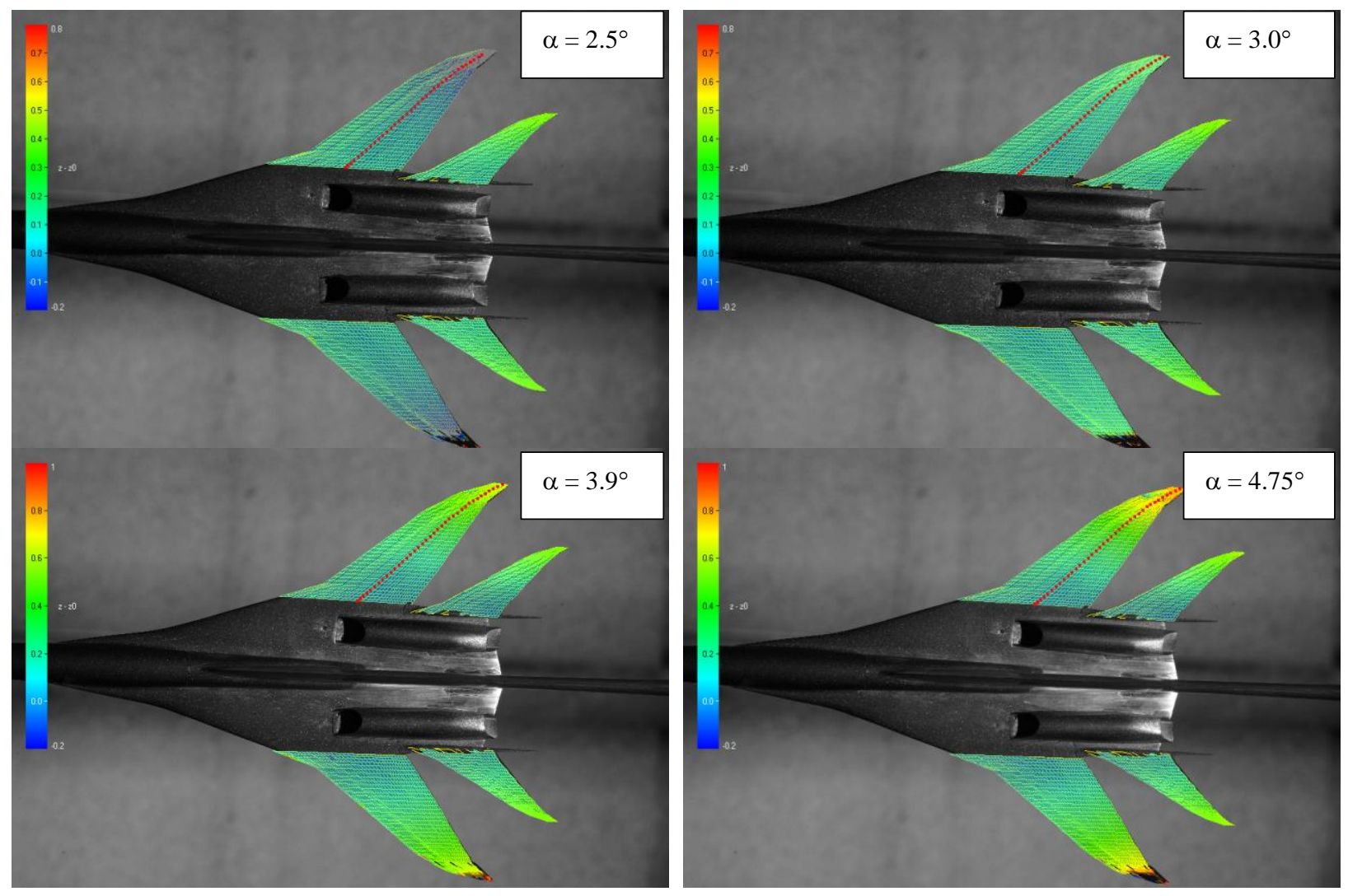

Figure 13. Images of Boom model with bending-data overlays.

The coordinates of the photogrammetry measurements were defined by the calibration plate. Any error in aligning the plate to the wind tunnel would produce a bias error in the corresponding angle and position measurements. Just as for the tunnel data system, gravity could not be used to establish zero pitch. Instead, the zeropitch angle was set by using a laser level and tape measure to position the plate as parallel to the sidewalls as possible - probably no better than $0.1^{\circ}$. The positions of the plate relative to the models were measured with the tape measure to an accuracy of about 1/16 in. These errors had little effect on the model bending and twist measurements because each measurement was the difference between wind-off and wind-on data, both of which included the same errors, which would cancel out. In contrast, errors in orienting the calibration plate would have a large effect on measurements of $\alpha$ (Figs. 16) because these measurements were absolute, not relative. The cameras were calibrated separately for measurements of the Performance and Boom models, and the calibration plate had to be re-installed at a different position for each model. This probably explains why the biases of the MDM measurements for the two models were not both in the same direction. However, the magnitude of the average offset of the MDM data for the Boom model $\left(0.5^{\circ}\right)$ is larger than the expected due to uncertainty in the angle of the calibration plate.

Uncertainty due to random errors in locating corresponding points in the images of both cameras were estimated by perturbing, one at a time, the image coordinates of each point used to compute each MDM quantity (bending, twist, $\alpha$ ) and observing the resulting perturbation, $\Delta_{i}$, in the computed values. If the uncertainty in locating each target is independent of the uncertainty in locating any other target, then the resulting uncertainty in each quantity due to target-location uncertainty is $\Delta=\sqrt{\sum_{1}^{n} \Delta_{i}^{2}}$, where $n$ is the number of targets upon which the measurement of that quantity depends. Table 1 summarizes the uncertainties in bending, twist, and angle of attack for each model due to a one-pixel uncertainty in locating rigid-body targets and flexible-body targets (i.e., grid nodes, where model bending was measured). 

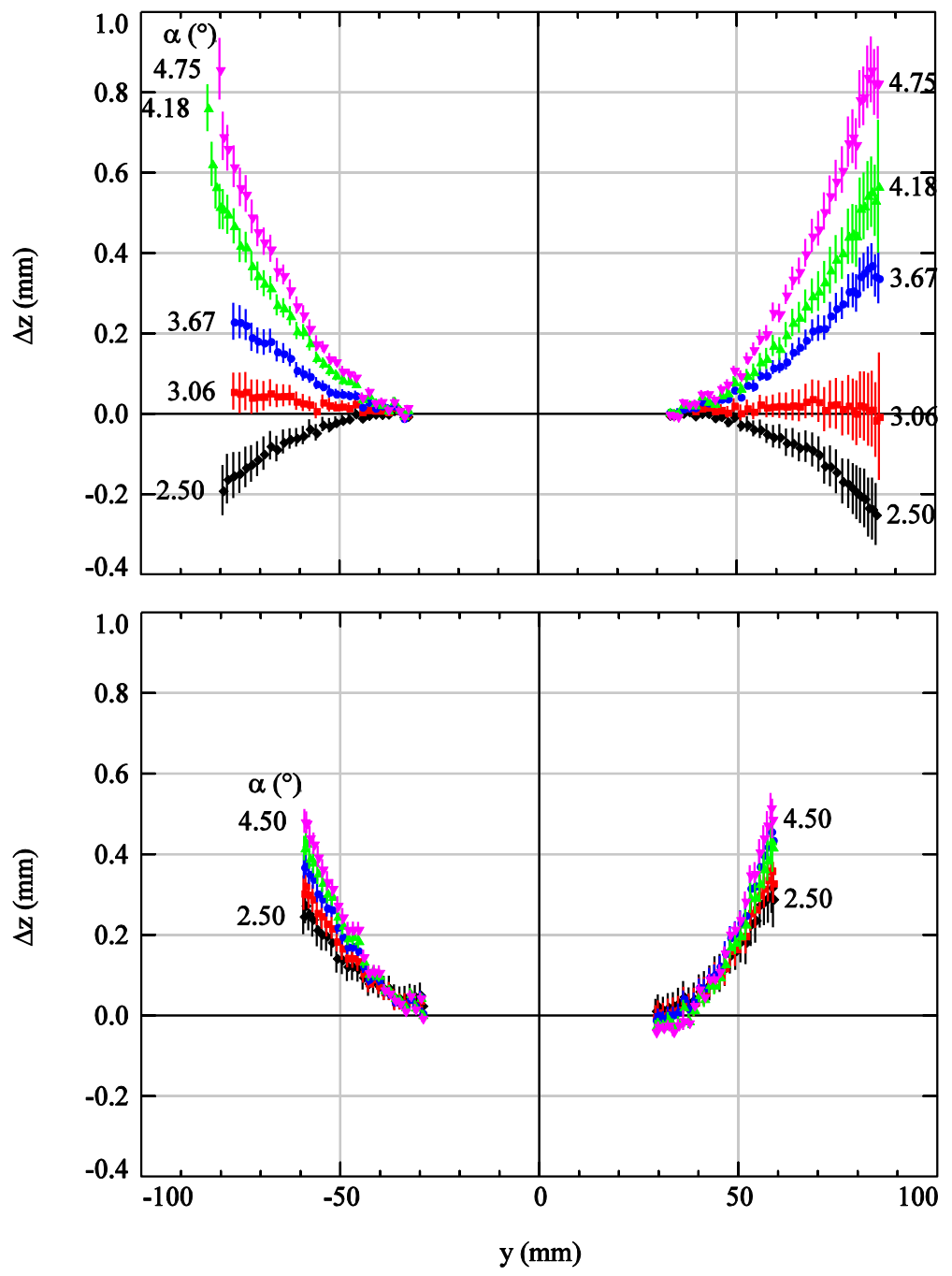

Figure 14. Bending versus span along wings (top) and tail (bottom) of Boom model. visible in wind-on images, they could be used to re-calibrated the cameras on an image-by-image basis to account for camera vibration or to restore calibration of a camera that was bumped out of alignment. Of course, the space coordinates of these targets must be accurately known. [We did not have the time or means in the present test to measure wall targets.] In addition, because the targets would not be in the region of interest, large depth-of-field would be required to assure adequate focus both at the model and the walls. This implies bright illumination.

An advantage of using a calibration plate is that it allows placing targets in the region of interest, which is desirable for DLT calibrations and relaxes depth-of-field requirements. However, by using a pinhole calibration instead of the DLT, the wall targets would be used only to compute the position and point angles of the cameras ("resection") and would not need to be in the region of interest. The use of the pinhole method requires a second calibration to establish the "internal orientation" of the cameras (focal length, principal point, and lens distortion). This can be quickly and easily accomplished in situ by acquiring images of a planar array of targets (for example, targets on a hand-held board) from many directions. This approach has been successfully demonstrated for MDM in subsequent tests in the Ames 11- by 11-Ft Transonic Wind Tunnel. 

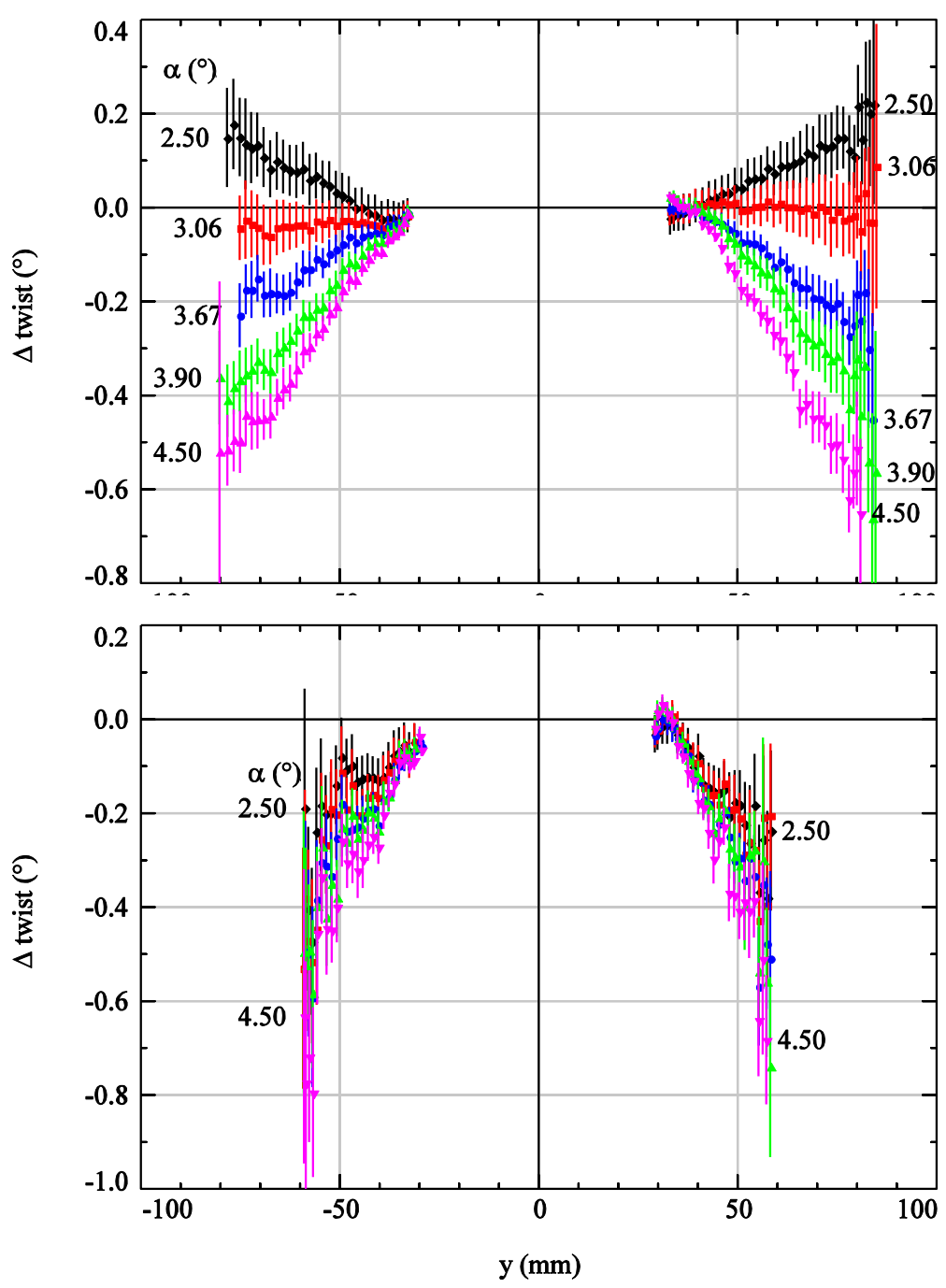

Figure 15. Twist due to bending vs span along wing (top) and tails (bottom) of Boom model.
The conventional targets were inadequate, both because there were too few of them and because the contrast with the speckle background was so low that the targets were sometimes hard to find. Thus the target-based bending and twist measurements were far inferior to the speckle-based measurements. The speckles, on the other hand, were nearly ideal. The lack of targets was especially severe on the rigid centerbody of the Boom model.

The time required to download the huge image files from the cameras to the computer seriously limited the rate at which MDM data could be acquired and would be unacceptable for most tests in a production wind tunnel. In addition, if photogrammetry is to be used to measure of angle of attack in a production wind tunnel, hardware and data-handling processes must be incorporated that yield measurements in at least near-real time.

\section{Conclusion}

Aeroelastic deformations of two sonic-boom models were successfully measured by stereo photogrammetry during tests in the NASA Ames 9- by 7-Ft Supersonic Wind Tunnel. The combination of speckles on the wing and tail surfaces and very high resolution cameras yielded essentially continuous measurements of bending and changes in twist everywhere on

the speckled surfaces. Elastic deformations smaller than $0.1 \mathrm{~mm}$ and changes in twist of less

than $0.05^{\circ}$ were resolved. By-products of the measurements were the instantaneous positions and attitudes of the models, including angle of attack. The principal source of uncertainty in the measurements was uncertainty in the alignment of a calibration plate with respect to the wind tunnel, which produced a uniform offset of the MDM angle measurements from measurements by the tunnel data system. A different calibration procedure was proposed that would eliminate this error.

Table 1. Uncertainties in bending, bending-induced twist, and angle of attack due to uncertainty of 1 pixel in locating targets in images.

\begin{tabular}{|l|c|c|c|c|}
\hline & \multicolumn{2}{|c|}{ Performance (run 1334 seq 9) } & \multicolumn{2}{c|}{ Boom (run 1805 seq 3) } \\
\hline Target type & Rigid-Body & Flexible-Body & Rigid-Body & Flexible-Body \\
\hline Bending near tip $(\mathrm{mm})$ & 1.32 & 0.17 & 1.09 & 0.17 \\
\hline Twist near tip $\left(^{\circ}\right)$ & 0.08 & 0.06 & 0.097 & 0.23 \\
\hline Angle of attack $\left(^{\circ}\right)$ & 0.09 & - & 0.049 & - \\
\hline
\end{tabular}




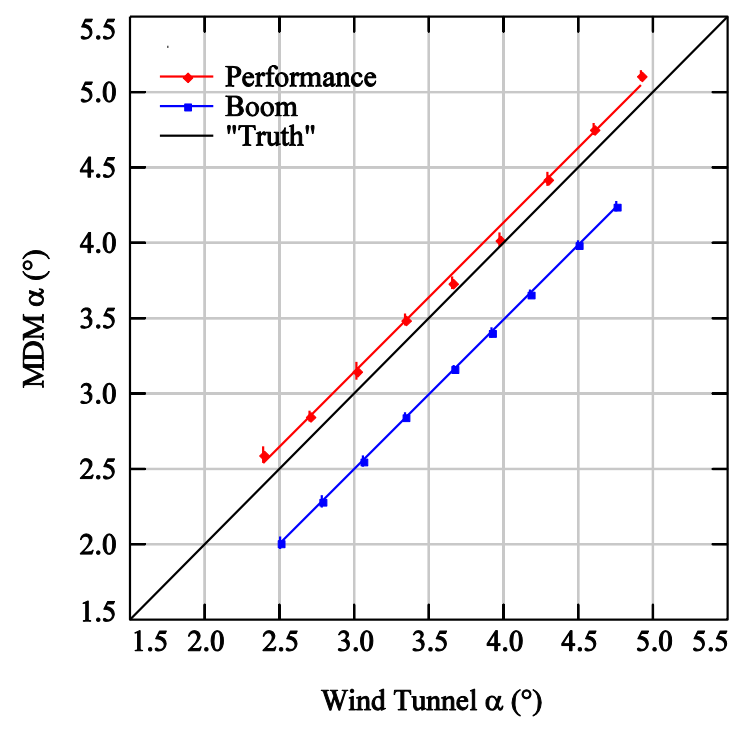

Figure 16. Comparison of angles of attack measured by MDM and SDS.

110229, Feb. 1996.

${ }^{7}$ Burner, A.W., Radeztsky, R.H., and Liu, T., "Videometric Applications in Wind Tunnels," Videometrics V, Proceedings of the International Society for Optical Engineering, Vol. 3174, San Diego, 1997, pp. 234-247.

${ }^{8}$ Burner, A.W., and Liu, T., "Videogrammetric Model Deformation Measurements Technique, Journal of Aircraft, Vol. 38, No. 4, July-Aug. 2001, pp. 745-754.

${ }^{9}$ Raffel, M., Willert, C., and Kompenhans, J., Particle Image Velocimetry, A Practical Guide, Springer, Berlin, 1998.

${ }^{10}$ Lucas, B.D., and Kanade, T., "An Iterative Image Registration Technique with an Application to Stereo Vision,”

Proceedings of the $7^{\text {th }}$ International Joint Conference on Artificial Intelligence (IJCAI), Aug. 24-28, 1981, Vancouver, British Columbia, Canada, pp. 674-679.

${ }^{11}$ Kirmse, T., "Model Deformation Measurements in DNW-NWB within the DLR Project ForMEx," ODAS 2007, $8^{\text {th }}$ ONERA-DLR Aerospace Symposium.

${ }^{12}$ Kushner, L.K., and Cassell, A., "Photogrammetry of a Hypersonic Inflatable Aerodynamic Decelerator," AIAA Paper 2013-1284 presented at AIAA Aerodynamic Decelerator Systems (ADS) Technology Conference, Mar. 25-28, 2013, Daytona Beach, FL.

${ }^{13}$ Li, L., "Photogrammetry Analysis of a Hypersonic Inflatable Aerodynamic Decelerator Structural Test Article," AE8900 MS Special Problems Report Space Systems Design Lab (SSDL) Guggenheim School of Aerospace Engineering Georgia Institute of Technology, Atlanta, GA, July 2013.

${ }^{14}$ Pallek, D., Butefisch, K.A., Quest, J., and Strudthoff, W., "Model Deformation Measurement in ETW Using the Moire Technique," $20^{\text {th }}$ International Congress on Instrumentation in Aerospace Simulation Facilities (ICIASF), Gottingen, Germany, Aug. 25-29 2003.

${ }^{15}$ Baumann, P.H., and Butefisch, "Measurement of Hinge Moment and Model Deformations in Wind Tunnels by Means of Moire Interferometry,” Optical Techniques in Fluid, Thermal, and Combustion Flows, SPIE, July 1995.

${ }^{16}$ Abdel-Aziz, Y.I. and Karara, H.M., "Direct Linear Transformation from comparator coordinates into object-space coordinates," Proceedings, Symposium on Close-Range Photogrammetry, American Society of Photogrammetry, Urbana, IL, Jan. 1971.

${ }^{17}$ Mikhail, E.M., Bethel, J.S., and McGlone, J.C., Introduction to Modern Photogrammetry, John Wiley and Sons, New York, 2001, pp. 92-95.

${ }^{18}$ Golub, G.H and Reinsch, C., "Singular value decomposition and least squares solutions," Numer. Math. Vol. 14, pp. 403420.

${ }^{19}$ Press, W.H. et al., Numerical Recipes, The Art of Scientific Computing, Cambridge University Press, Cambridge, 1986, pp 523-528. 